\title{
Orale Antikoagulation bei Vorhofflimmern reduziert das Demenzrisiko
}

Fragestellung: Es besteht ein Zusammenhang zwischen Vorhofflimmern und Demenz. Kann durch eine orale Antikoagulation das Risiko einer Demenz bei Patienten mit Vorhofflimmern reduziert werden?

Hintergrund: Es gibt viele epidemiologische Studien, die einen eindeutigen Zusammenhang zwischen Vorhofflimmern und der Entwicklung einer Demenz belegt haben. Dieser Zusammenhang besteht unabhängig davon, dass Patienten mit Vorhofflimmern ein erhöhtes Schlaganfallrisiko haben.

Die Demenz kann entweder Folge eines erlittenen Schlaganfalls sein oder auch durch Mikroembolien und den daraus resultierenden Mikroinfarkten bedingt sein. Erste kleinere Studien hatten einen Hinweis darauf gegeben, dass eine Antikoagulation mit Vitamin-K-Antagonisten (VKA) oder NichtVitamin-K-abhängigen oralen Antikoagulanzien (NOAK) das Risiko einer Demenz reduzieren kann.

Patienten und Methodik: Es handelt sich um eine retrospektive Studie, die auf das nationale Gesundheitsregister in Schweden zurückgreift. Das Register dokumentiert alle Krankenhausaufnahmen seit 1987. Seit 2005 werden auch alle verschreibungspflichtigen Medikamente registriert. Im Rahmen der Studie wurden alle Patienten erfasst, die zwischen 2006 und 2014 die Diagnose Vorhofflim-

Friberg L, Rosenqvist M. Less dementia with oral anticoagulation in atrial fibrillation. Eur Heart J 2017. doi: 10.1093/ eurheartj/ehx579 [Epub ahead of print] mern erhielten. Das waren in diesem Zeitraum in Schweden 456.960 Patienten. Bei 12.854 Patienten bestand zum Zeitpunkt der Diagnosestellung Vorhofflimmern bereits eine Demenz. Diese
Patienten wurden aus der Studie ausgeschlossen. In der Studie wurden die Daten von 444.106 Patienten ausgewertet. Während des Beobachtungszeitraums wurde bei 26.210 Patienten die Diagnose Demenz neu gestellt. 241.160 Patienten (54,3\%) erhielten keine oralen Antikoagulanzien, 190.570 Patienten $(42,9 \%)$ bekamen den VKA Warfarin und 12.916 Patienten $(2,9 \%)$ ein NOAK.

Ergebnisse: Die Patienten waren bei Einschluss in die Analyse im Mittel 74 Jahre alt. $20 \%$ hatten bereits in der Vorgeschichte einen Schlaganfall oder eine transitorische ischämische Attacke (TIA) erlitten. Patienten, die eine Demenz entwickelten, waren mit einem mittleren Alter von 81 Jahren älter als die Patienten, die keine Demenz entwickelten (74 Jahre). Die wichtigsten Prädiktoren für die Entwicklung einer Demenz waren zunehmendes Lebensalter, eine Parkinson-Erkrankung und Alkoholmissbrauch.

Die Autoren führten ein Propensity-Score-Matching durch und entwickelten zwei vergleichbare Kohorten von jeweils 80.948 Patienten mit und ohne orale Antikoagulation. Antikoagulierte Patienten hatten ein um 29\% niedrigeres Risiko, eine Demenz zu entwickeln, als nicht antikoagulierte Patienten. Wurden die Patienten analysiert, die im Verlauf ihre orale Antikoagulanzien tatsächlich einnahmen, ergab sich eine $48 \%$ ige Risikoreduktion zugunsten der oralen Antikoagulation. Der Vergleich zwischen NOAK und Warfarin zeigte keinen Unterschied.

Schlussfolgerungen: In einer großen Registerstudie in Schweden zeigt sich, dass Patienten mit Vorhofflimmern, die oral antikoaguliert werden, ein geringeres Risiko haben, eine Demenz zu entwickeln.

\section{Die statistischen Zusammenhänge müssen nicht unbedingt kausal sein}

Diese große Studie aus Schweden mit 444.106 Patienten und 1,5 Millionen Patientenjahren zeigt, dass eine orale Antikoagulation bei Patienten mit Vorhofflimmern offenbar das Risiko einer Demenz reduziert. Dies gilt sowohl für eine Auswertung zum Zeitpunkt des Einschlusses in die Studie als auch für eine zweite Analyse, bei der nur Patienten berücksichtigt wurden, die eine orale Antikoagulation über längere Zeit erhielten.

Die Ergebnisse waren für alle wesentlichen Untergruppen von Begleiterkrankungen und vaskulären Risikofaktoren identisch. Wie bei anderen Registerstudien muss allerdings berücksichtigt werden, dass es sich hier um statistische Zusammenhänge handelt, die nicht notwendigerweise im vollen Umfang kausal sein müssen. So mag es gute Gründe geben, warum bestimmte Patienten mit Vorhofflimmern nicht antikoaguliert wurden, beispielsweise weil sie ein erhöhtes Blutungsrisiko hatten. Dessen ungeachtet verdichtet sich jetzt durch viele epidemiologische Studien, dass mit hoher Wahrscheinlichkeit eine orale Antikoagulation bei Patienten mit Vorhofflimmern das Risiko der Entwicklung einer Demenz reduziert.

In der vorliegenden Studie waren die Untersucher jedoch nicht in der Lage, zwischen degenerativer und vaskulärer Demenz zu unterscheiden. In einer holländischen Studie hatte sich gezeigt, dass Vorhofflimmern sowohl das Risiko einer Alzheimer-Demenz als auch das Risiko einer vaskulären Demenz erhöht. 